\section{A new pathway for CYLD}

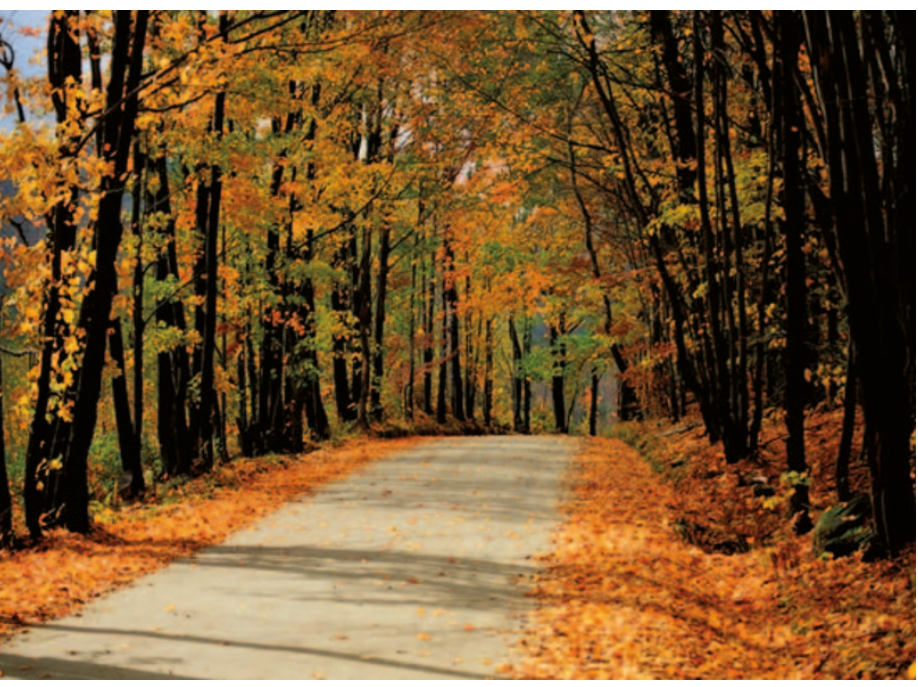

In humans, the loss of both $C Y L D$ alleles causes the development of benign and disfiguring skin tumours called cylindromas. Using skin cells from Cyld ${ }^{-1-}$ mice, Reinhard Fässler and colleagues show that the deubiquitylase CYLD, in addition to its known effects on the NFKB pathway, can control the nuclear translocation of the nuclear factor $\kappa \mathrm{B}(\mathrm{NF \kappa B})$ coactivator BCL3.

The authors showed that the Cyld ${ }^{-1-}$ mice they generated are more prone to DMBA (7,12-dimethylbenz(a)anthracene) and TPA (12-O-tetradecanoylphorbol-13acetate)-induced skin tumours than wild-type mice. Bromodeoxyuridine incorporation and Ki67 expression indicated increased proliferation in Cyld ${ }^{-/-}$tumours compared with wild-type tumours, but no difference in the rate of apoptosis was observed. In addition, Cyld ${ }^{-1-}$ tumours showed increased expression of cyclin D1 (encoded by Ccnd1), and increased cyclin D1 expression was also observed in isolated primary Cyld ${ }^{-/-}$keratinocytes treated with TPA or UVB light.

Is there a link between CYLD loss and cyclin D1 expression? Using reporter assays, the authors showed that TPA or UVB activates the Ccnd 1 promoter in Cyld ${ }^{-/-}$keratinocytes in an NFKB-dependent manner, indicating that CYLD is a negative regulator of this pathway. CYLD has been implicated in the inhibition of tumour-necrosis factor- $\alpha$ (TNF $\alpha$ )-induced activation of the NFкB p65/p50 heterodimer, in part through the stabilization of the NFאB inhibitor I $\mathrm{B} \alpha$. However, TPA treatment failed to increase $\mathrm{p} 65 / \mathrm{p} 50$ dependent transcription, indicating that TPA triggers NFKB activity in an $I \kappa B a-i n d e p e n d e n t$ manner in keratinocytes.

So, which NFkB family member(s) regulates cyclin D1 expression? Transfection of Cyld ${ }^{-/-}$and wild-type keratinocytes with the Ccnd 1 promoter reporter construct and various $\mathrm{NF} \kappa \mathrm{B}$ family members showed that $\mathrm{p} 50$ or $\mathrm{p} 52$, as well as the coactivator BCL3, activated the promoter in the absence of CYLD. Co-immunoprecipitation analyses showed that CYLD is associated with BCL3 in keratinocytes in response to TPA, and TPA or UVB treatment increased nuclear translocation of BCL3 in Cyld - $^{-1}$ keratinocytes, DMBA/TPA-induced Cyld ${ }^{-1-}$ tumours and human cylindromas. Furthermore, chromatin immunoprecipitation showed that in Cyld $^{-1-}$ keratinocytes, TPA treatment recruits BCL 3 and $\mathrm{p} 50$ or p52, but not p65, to the $C$ cnd 1 promoter.

Is the deubiquitylating activity of CYLD required to prevent BCL3 nuclear accumulation? TPA treatment significantly increased polyubiquitylation of BCL3 in Cyld $^{-1-}$ keratinocytes, and CYLD removed lysine-63-linked polyubiquitin chains from BCL3 in vivo - ubiquitin chains at this site usually serve as docking sites for other proteins. Catalytically inactive CYLD was unable to prevent BCL3 nuclear accumulation and activation of the Cond 1 promoter. These data indicate that deubiquitylation by CYLD is necessary to prevent BCL3 nuclear accumulation.

Previous data have shown reduced CYLD expression in human kidney, liver and cervical tumours, and the authors found reduced or absent CYLD expression in human basal-cell and squamous-cell carcinomas. Therefore, the mechanism of CYLD-mediated suppression of NFKB signalling proposed by Fässler and colleagues might be important in several tumour types.

Sarah Seton-Rogers

ORIGINAL RESEARCH PAPER Massoumi, R. et al. Cyld inhibits tumor cell proliferation by blocking Bcl-3-dependent NF-KB signaling. Cell 125, 665-677 (2006)

\section{RESEARCH HIGHLIGHTS ADVISORS}

AVI ASHKENAZI Genentech, Inc. South San Francisco, CA, USA JOSE BASELGA

Vall d'Hebron University Hospital,

Barcelona, Spain

ANTON BERNS

Netherlands Cancer Institute

Amsterdam, The Netherlands
MARIA BLASCO

Spanish National Cancer Centre (CNIO), Madrid, Spain RON DEPINHO Harvard Medical School, Boston, MA, USA GLENN DRANOFF

Dana-Farber Cancer Institute, Boston, MA, USA
RAKESH JAIN

Massachusetts General Hospital, Boston, MA, USA CHRISTOPH LENGAUER Novartis Institute for Biomedical Research Inc.. Cambridge, MA, USA LANCE LIOTTA National Cancer Institute, Bethesda, MD, USA
JOHN D. POTTER Fred Hutchinson Cancer Research Center, Seattle, WA, USA DAVID SIDRANSKY Johns Hopkins University School of Medicine, Baltimore, MD, USA
BERT VOGELSTEIN

The Sidney Kimmel Comprehensive Cancer Center, Baltimore, MD, USA ROBERT WEINBERG

Whitehead Institute for Biomedical Research, Cambridge, MA, USA ZENA WERB University of California at San Francisco, CA, USA 